\title{
Facial botryomycosis-like pyoderma in an HIV-infected patient: remission after initiation of darunavir and raltegravir
}

\author{
Walter de Araujo Eyer-Silva ${ }^{[1]}$, Guilherme Almeida Rosa da Silva ${ }^{[1]}$, \\ Fernando Raphael de Almeida Ferry ${ }^{[1]}$ \\ and Jorge Francisco da Cunha Pinto ${ }^{[1]}$
}

[1]. Centro de Ciências Biológicas e da Saúde, Hospital Universitário Gaffrée e Guinle, Universidade Federal do Estado do Rio de Janeiro, RJ, Brasil.

\begin{abstract}
Botryomycosis is an uncommon, chronic, suppurative, bacterial infection that primarily affects the skin and subcutaneous tissues. It has long been associated with defects of cellular immunity. We report a 28 -year-old woman who presented with a chronic, ulcerated lesion with draining sinuses in the right malar region. Predisposing factors were HIV infection with poor immunological control, alcoholism, and a previous trauma to the right cheek. Several courses of antimicrobial therapy provided only partial and temporary remission. Complete clinical remission was only achieved 5 years later when a novel antiretroviral regimen composed of darunavir and raltegravir was initiated.
\end{abstract}

Keywords: AIDS. Botryomycosis. HIV infection.

\section{INTRODUCTION}

Botryomycosis is an uncommon, chronic, suppurative, bacterial infection that affects the skin, subcutaneous tissues, and, occasionally, visceral organs, mainly the lungs ${ }^{1}$. Cutaneous botryomycosis presents with nodules, plaques, ulcers, multiple draining sinuses, fistulas, cysts, and irregular masses. Extension to soft tissues, muscles, and bones may occur ${ }^{2}$. Clinically and pathologically, botryomycosis often mimics mycetoma, both actinomycotic and eumycotic. Staphylococcus aureus is by far the most frequently implicated agent ${ }^{1}$. Several other bacterial agents that are part of the skin microbiota have also been incriminated as the cause of botryomycosis, and it can also be polymicrobial ${ }^{3}$. Bacteria are most commonly inoculated through a preceding trauma. Histopathologically, it is characterized by the presence of grains that represent clusters of non-filamentous bacteria, cells, and debris. Grains are often visible with the naked eye and are similar to those of mycetoma ${ }^{4}$.

Botryomycosis is exceedingly rare in the head and neck, and has long been associated with immunological abnormalities ${ }^{2,5,6}$. We wish to report an HIV-infected patient who developed facial botryomycosis-like pyoderma, in whom clinical remission was only achieved 5 years later when a novel antiretroviral regimen composed of darunavir and raltegravir was instituted.

Corresponding author: Prof. Walter de Araujo Eyer Silva

e-mail: walter.eyer@ig.com.br

Received 24 February 2016

Accepted 18 October 2017

\section{CASE REPORT}

A 28-year-old woman presented in June 2010 with a nonhealing, ulcerated lesion with draining sinus in the right buccal region. The lesion was painless and intermittently drained a microgranular yellowish exudate (Figure 1A). There was no extension to the oral mucosa and no proximity to any bony tissue. The patient was afebrile and informed that the lesion emerged at the precise site of a trauma that occurred 5 years earlier.

She was diagnosed with HIV infection 6 years prior during her prenatal checkup, and has since been followed up at the local municipal human immunodeficiency virus/ acquired immunodeficiency syndrome (HIV/AIDS) program in Northwestern Rio de Janeiro State. The clinical, epidemiological, and virological features of HIV infection in the region have been previously reported ${ }^{7}$. Between December 2009 and June 2010, she resided in another city and first noted the right cheek lesion. At this point, the cluster of differentiation 4 (CD4) cell count was $461 / \mathrm{mm}^{3}$ and the plasma HIV viral load was 2,431 copies/ml (3.38 log 10). Serological assays for syphilis were negative. In the preceding 3 years, she had been offered multiple antiretroviral regimens that included combinations of nucleoside and non-nucleoside reverse transcriptase inhibitors, as well as the protease inhibitors atazanavir and lopinavir. However, she was poorly adherent to treatment and complained of intolerance to multiple antiretroviral agents. Frequent episodes of alcohol abuse were also recorded. 
Gram staining of the draining material showed Grampositive cocci that occurred singly, as well as in pairs, short chains, and grapelike clusters. Small, Gram-negative pleomorphic bacilli were also present. The samples were negative for acid-fast bacilli and fungi. The presence of grains was not reported by the laboratory. Bacterial, mycobacterial, and fungal cultures of the draining material were requested before initiation of antimicrobial therapy. There was no growth of fungi or mycobacteria after several weeks. Bacterial cultures yielded S. aureus and Acinetobacter spp., both of which were resistant only to penicillin.

Between 2010 and 2014, the patient experienced only partial and temporary improvements after several courses of 3 to 6 weeks of antimicrobial therapy with either a quinolone or a cephalosporin agent. This partial remission, however, was soon followed by relapse, spread to adjacent tissues, and formation of draining sinuses and fistulas. Some previously affected areas healed with scarring (Figure 1B/C). The patient refused surgical debridement and excision. Repeated cultures of the draining material were negative for fungi and mycobacteria. No further samples of the exudate could be obtained for additional microbiological analyses. Repeated CD4 cell count measurements showed a sharp drop to 99 cells $/ \mathrm{mm}^{3}$ (July 2012) and 119/ $/ \mathrm{mm}^{3}$ (March 2013). An undetectable HIV plasma viral load measurement was never recorded (range 3.3-5.3 log).

An HIV genotyping study was performed to investigate the presence of mutations associated with reduced susceptibility to HIV reverse transcriptase and protease inhibitors. No such mutations were recorded, a pattern suggestive of poor adherence to treatment. A decision was made to initiate a novel antiretroviral regimen composed only of the protease inhibitor darunavir (along with a booster dose of ritonavir) and the integrase inhibitor raltegravir. No reverse transcriptase inhibitors were added. The new regimen was started in May 2015. Following initiation of the new treatment regimen, the malar lesion started to improve, and a total remission was observed a few months later (Figure 1). The patient reported that the darunavir-raltegravir regimen was better tolerated. The CD4 cell count showed a recovery to 400 cells $/ \mathrm{mm}^{3}$ in 6 months and 576/ $\mathrm{mm}^{3}$ after 1 year. The plasma viral load dropped to 915 copies/ $\mathrm{ml}(2.9 \mathrm{log})$ in 6 months and to below detection limits 1 year later. The patient has now completed 1 year of full remission, with no relapse of the facial lesion (Figure 1D).

\section{Informed consent}

Informed consent was obtained from the patient for publication of the case.

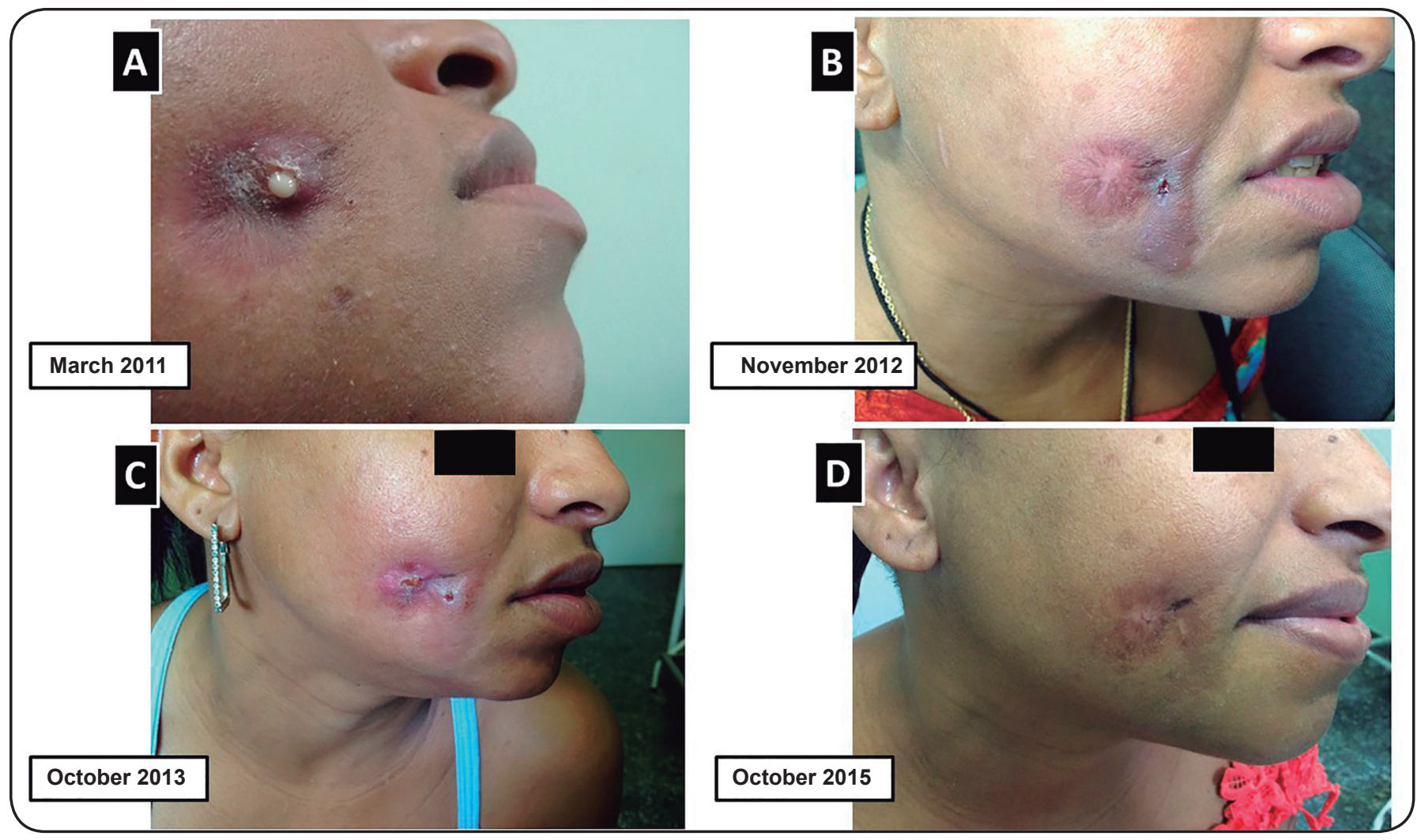

FIGURE 1. Five-year follow-up of botryomycosis-like pyoderma on the right malar region of a 28 -year-old HIV-infected woman between 2011 and 2015. (A): March 2011: a non-healing, ulcerated lesion draining a yellowish exudate. (B): November 2012: partial and temporary remission after several weeks of antimicrobial therapy. Spread to adjacent rostral tissues is observed. The original site healed with scarring. (C): October 2013: relapse of botryomycosis-like pyoderma at the original and rostral sites. At this point, no exudate was available for additional microbiological analyses. (D): October 2015: full remission after initiation of the novel antiretroviral combination. The patient has completed 1 year of follow-up without relapse. 


\section{DISCUSSION}

To date, approximately 200 cases of botryomycosis have been published in the medical literature ${ }^{4}$. Botryomycosis was first described by Bollinger in 1870 in the lung of a horse $e^{\text {apud2, }}$. The term botryomycosis was coined by Rivolta in $1884^{\text {apud } 2,4}$; it is a combination of the Greek words botrys (bunch of grapes) and mycosis, as the disease was of presumed to have a fungal origin. Although a misnomer, the term continues to be used in the medical literature for historical reasons. The first human integumentary and visceral cases were described in 1903 and 1913 by Spitz and Opie, respectively. In 1919, Magrou established its bacterial origin by isolating $S$. aureus and reproducing the disease in guinea pigs ${ }^{\text {apud } 2,4}$

Neafie \& Marty ${ }^{1}$ proposed that the diagnosis of botryomycosis can be established by I) identifying non-filamentous bacteria in granules in pus from draining sinuses; II) culturing the bacterium from ulcers or exudates in patients with the clinical findings of botryomycosis, or III) recognizing non-filamentous bacteria in granules in biopsy or autopsy specimens ${ }^{1}$. In the present case, the diagnosis of botryomycosis-like pyoderma was established based upon the failure to recognize fungal and branching organisms on direct staining, the negative cultures for fungi and mycobacteria, the presence of grapelike Gram-positive cocci and Gram-negative pleomorphic bacilli on staining, the growth of $S$. aureus and Acinetobacter sp. on culture, and the chronic cutaneous lesions compatible with botryomycosis. No biopsy was available to rule out the presence of fungal or branching organisms, as well as of grains, on tissue sections. However, we believe that the full remission achieved following initiation of darunavir and raltegravir, without any antibacterial therapy, strongly favored botryomycosis, as opposed to actinomycotic or eumycotic mycetoma, as the former is strongly associated with defects in cellular immunity. At least one previous botryomycosis report has documented the simultaneous participation of $S$. aureus and a species of Acinetobacter ${ }^{3}$.

Our patient's cutaneous disease began in 2010 and remained active for 5 years. At presentation, the differential diagnosis included actinomycetoma, eumycetoma, sporotrichosis, paracoccidioidomycosis, cutaneous leishmaniasis, mycobacterial disease, and botryomycosis. She had several predisposing factors to botryomycosis: previous trauma, alcoholism, and HIV infection with poor immunological and virological control. A literature review on botryomycosis revealed that many patients had immunological abnormalities ${ }^{5}$. The resolution of our patient's lesion after initiation of a novel antiretroviral regimen consisting of darunavir and raltegravir was associated with significant immunological recovery. In fact, some dual antiretroviral treatment strategies have been proven to combine antiviral activity with improved tolerability ${ }^{8}$.

Botryomycosis has long been reported as a complication of HIV infection since the early years of the epidemic. Among HIV-infected patients, the presentation may be atypical and the diagnosis may be challenging 9 . Atypical lesions resembling prurigo nodularis ${ }^{10}$, herpes-like lesions ${ }^{11}$, treatment failure of multiple antimicrobial agents ${ }^{9}$, and the association of $S$. aureus with cutaneous Pneumocystis jirovecii ${ }^{12}$ have been reported.
The pathogenesis of this insidious infection is still unclear. It most probably represents a delicate balance between the number of inoculated organisms, the virulence of the infecting agent(s), and the host's predisposing factors. Successful treatment of botryomycosis depends on several factors, such as the immune status of the host, the causative agent(s), and the location and extent of the lesion(s). The mainstay of therapy is appropriate antibiotics guided according to bacterial resistance patterns. Surgery is generally reserved for diagnostic purposes or recalcitrant disease. A diagnosis of botryomycosis should always alert the clinicians to the possibility of an underlying immunological disorder.

\section{Conflict of interest}

The authors declare there is no conflict of interest.

\section{REFERENCES}

1. Neafie RC, Marty AM. Unusual Infections in Humans. Clin Microb Rev. 1993;6(1):34-56.

2. Yencha MW, Karakla DW. Cutaneous botryomycosis of the cervicofacial region. Head Neck. 2001;23(7):594-8.

3. Simantov A, Chosidow O, Fraitag S, Feuilhade de Chauvin M, Prost C, Ravisse P, et al. Disseminated cutaneous botryomycosis - an unexpected diagnosis after 20-years' duration. Clin Exp Dermatol. 1994;19(3):259-61.

4. Padilla-Desgarennes C, Vásquez-González D, Bonifaz A. Botryomycosis. Clin Dermatol. 2012;30:397-402.

5. Brunken RC, Lichon-Chao N, van der Broek H. Immunologic abnormalities in botryomycosis. A case report with review of the literature. J Am Acad Dermatol. 1983;9(3):428-34.

6. Guttal KS, Naikmasur VG, Satyabodh K. Botryomycosis of the orofacial region. Br J Oral Maxillofac Surg. 2008;46(5):424-5.

7. Eyer-Silva WA, Couto-Fernandez JC, Morgado MG. Molecular epidemiology of HIV type 1 in inner Rio de Janeiro State, Brazil. AIDS Res Hum Retroviruses. 2007;23(2):303-8.

8. Baril JG, Angel JB, Gill MJ, Gathe J, Cahn P, van Wyk J, et al. Dual therapy strategies for the management of patients infected with HIV: a systematic review of current evidence in ARV-naïve or ARV-experienced, virologically suppressed patients. Plos One. 2016;11(2):e0148231.

9. Rubenstein E, Krulig E, Cardenas V, Kerdel FA. Botryomycosislike pyoderma in the genital region of a human immunodeficiency virus (HIV)-positive man successfully treated with dapsone. Int J Dermatol. 2010;49(7):842-3.

10. Templet JT, Straub R, Ko C. Botryomycosis presenting as pruritic papules in a human immunodeficiency virus-positive patient. Cutis. 2007;80(1):45-7.

11. de Vries HJ, van Noesel CJ, Hoekzema R, Hulsebosch HJ. Botryomycosis in an HIV-positive subject. J Eur Acad Dermatol Venerol. 2003;17(1):87-90.

12. Saadat P, Ram R, Sohrabian S, Vadmal MS. Botryomycosis caused by Staphylococcus aureus and Pneumocystis carinii in a patient with acquired immunodeficiency disease. Clin Exp Dermatol 2008;33(3):266-9. 